\title{
Lunar In Situ Materials-Based Surface Structure Technology Development Efforts at NASA/MSFC
}

\author{
M. R. Fiske ${ }^{1}$, W. McGregor ${ }^{2}$, R. Pope ${ }^{2}$, C. A. McLemore ${ }^{3}$, R. Kaul ${ }^{3}$, G. Smithers ${ }^{3}$, \\ E. Ethridge ${ }^{3}, \mathrm{H}$. Toutanji ${ }^{4}$ \\ ${ }^{1} J a c o b s$ Sverdrup/Marshall Space Flight Center, Huntsville, AL 35812 \\ ${ }^{2}$ Qualis Corporation/Marshall Space Flight Center, Huntsville, AL 35812 \\ ${ }^{3}$ NASA/Marshall Space Flight Center, Huntsville, AL 35812 \\ ${ }^{4}$ Civil Engineering Department, University of Alabama in Huntsville, Huntsville, AL 35805 \\ '256-544-6456, Michael.R.Fiske@.nasa.gov
}

\begin{abstract}
For long-duration missions on other planetary bodies, the use of in situ materials will become increasingly critical. As man's presence on these bodies expands, so must the structures to accommodate them, including habitats, laboratories, berms, radiation shielding for surface reactors, garages, solar storm shelters, greenhouses, etc. The use of in situ materials will significantly offset required launch upmass and volume issues. Under the auspices of the In Situ Fabrication \& Repair (ISFR) Program at NASA/Marshall Space Flight Center (MSFC), the Surface Structures project has been developing materials and construction technologies to support development of these in situ structures. This paper will report on the development of several of these technologies at MSFC's Prototype Development Laboratory (PDL). These technologies include, but are not limited to, development of extruded concrete and inflatable concrete dome technologies based on waterless and water-based concretes, development of regolith-based blocks with potential radiation shielding binders including polyurethane and polyethylene, pressure regulation systems for inflatable structures, production of glass fibers and rebar derived from molten lunar regolith simulant, development of regolithbag structures, and others, including automation design issues. Results to date and lessons learned will be presented, along with recommendations for future activities.
\end{abstract}

Keywords: In Situ, Lunar, Surface Structures.

PACS: 01:50.My, 07.10-h.

\section{INTRODUCTION}

Habitat manufacturing and assembly technologies that incorporate in situ resources provide options for autonomous, affordable, pre-positioned habitat environments with radiation shielding features and protection from micrometeorites, exhaust plume debris, and other hazards. This is important because gamma and particle radiation constitute a serious but reducible threat to long-term survival in space environments. Structures will constitute the primary mass element of the Moon or Mars habitation equipment set. The ability to use in situ material to construct these structures will provide a benefit in the reduction of upmass that would otherwise make the long term Moon and Mars habitation cost prohibitive. The ability to fabricate structures in situ brings with it the ability to repair these structures, which allows for self sufficiency necessary for long duration habitation.

The Exploration Systems Mission Directorate (ESMD) champions an evolutionary acquisition approach wherein the needs pull the technology research and development efforts. In compliance with this approach, the ISFR Habitat Structures activity has defined a Lunar Habitat capability and a Martian Habitat capability. Technologies are evaluated against the needs associated with these capabilities. Technology maturation activities are then phased 
according to resources to yield deliverables in the form of concepts, derived requirements, analyses, and demonstrations (Bodiford, et al., 2005). NASA's 1997 Habitats and Surface Construction Technology and Development Roadmap (Cohen \& Kennedy, 1997) identified three broad classes of space and surface habitats and the means of constructing them, as shown in Table 1.

TABLE 1. NASA Planetary Surface Habitat Classifications (Cohen \& Kennedy, 1997).

\begin{tabular}{|c|l|l|}
\hline $\begin{array}{c}\text { Class of } \\
\text { Construction }\end{array}$ & \multicolumn{1}{|c|}{ Title } & \multicolumn{1}{c|}{ Characteristics } \\
\hline I & Pre-Integrated & $\begin{array}{l}\text { Completely built and integrated on Earth before launch. Lands on surface and } \\
\text { stays in one place }\end{array}$ \\
\hline II & Deployable & $\begin{array}{l}\text { Completely built on Earth but may be integrated, assembled, deployed, erected, } \\
\text { inflated, moved, or reconfigured on the Lunar/planetary surface }\end{array}$ \\
\hline III & $\begin{array}{l}\text { In Situ Resource } \\
\text { Utilization (ISRU) }\end{array}$ & $\begin{array}{l}\text { May be built on Earth, but incorporates in situ materials on surface, or primary } \\
\text { structure may use in situ construction }\end{array}$ \\
\hline
\end{tabular}

The original scope of this effort addressed the approach for technology development of Class III, semi-automated, integrated habitat structure construction technologies that would lead up to the delivery of a subscale Technology Readiness Level 6 (TRL 6) demonstration targeted for FY14. However, at a Technical Interchange Meeting (TIM) held at MSFC in August 2005, this scope was increased to include a broader range of surface structure applications and associated construction technologies. These applications include berms for protection from exhaust plume impingement, unpressurized shelters for protection from the micrometeorite, thermal, and radiation environments, and greenhouses. In addition, reactor shields for containment of radiation from surface-based nuclear power plants and supplemental protective structures for Class I and II habitats were added to our technology portfolio. Of course, the development of Class III habitat structures, one example of which is shown in Figure 1, remained as the focus of our technology development efforts.

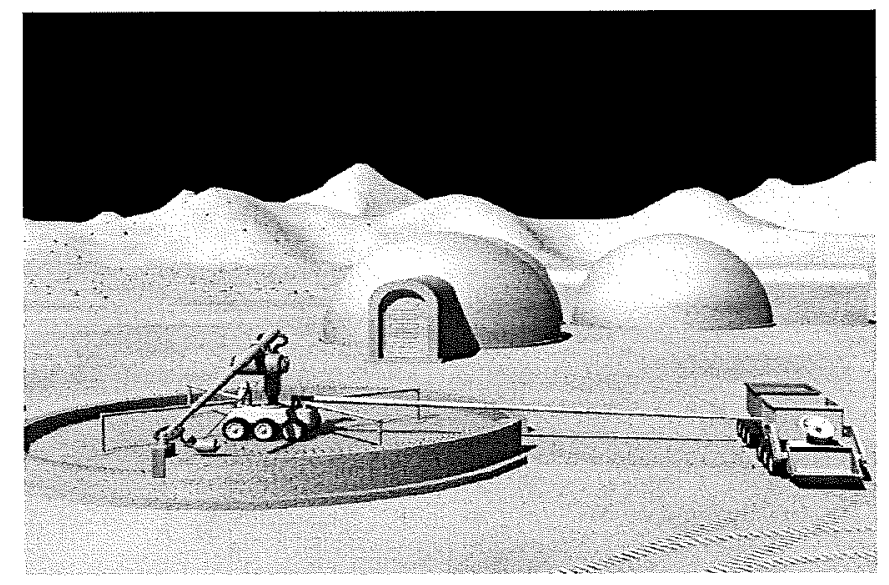

FIGURE 1. Lunar Habitat Concept Based on Extruded Concrete Process.

\section{CURRENT MSFC DEVELOPMENT ACTIVITIES}

MSFC is currently sponsoring a significant effort aimed at developing lunar in situ materials based on "waterless" concrete (Toutanji, Fiske and Bodiford, 2006). An associated effort at MSFC is to test these materials with respect to radiation mitigation, micrometeorite resistance, and resistance to larger, slower-traveling bits of regolith impinging on the surface as a result of nearby launch and landing activities. Figure 2 shows a 0.5 -in. cube of sulfur/JSC-1 concrete with approximately $20 \%$ silica added as filler after being impacted by a 1-mm aluminum sphere traveling at approximately $6 \mathrm{~km} / \mathrm{sec}$. As can be seen, the sample withstood this impact quite well, although this was significantly slower than the average speed of a micrometeorite, which is $20 \mathrm{~km} / \mathrm{sec}$. 


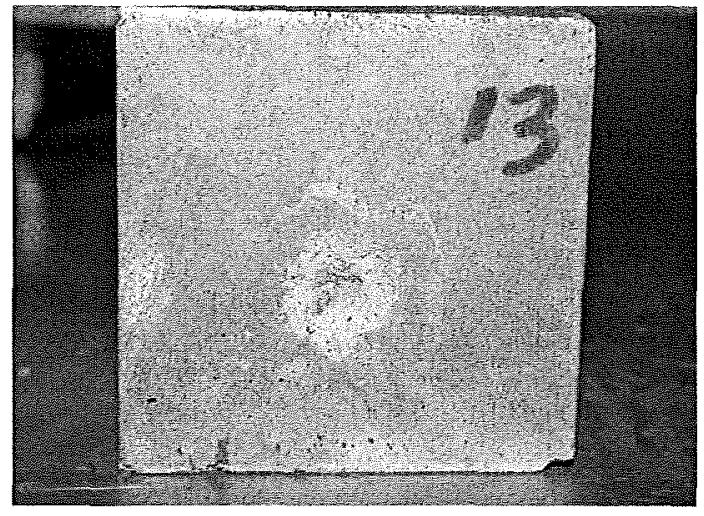

FIGURE 2. Sulfur Concrete Sample after Hypervelocity Impact Testing.

Another associated concrete development effort at MSFC is related to the actual processes used to make concrete structures in near net shapes. Under agreement with the University of Southern California (USC), an extruded concrete system (Contour Crafting, shown in Figure 3) process is being developed for possible application to the Moon and Mars (Khoshnevis, et al., 2005). The original system delivered by USC to MSFC is shown in the upper left of Figure 3, along with various concrete shapes this system has been able to manufacture. The upper right of Figure 3 shows the system after significant modifications, including:

- Incorporation of limit/home switches

- Script file modifications

- Fabrication and testing of new extrusion nozzles and a nozzle "shutter"

- Evaluation and incorporation of "Y" axis modifications to allow fabrication of more complex geometries.

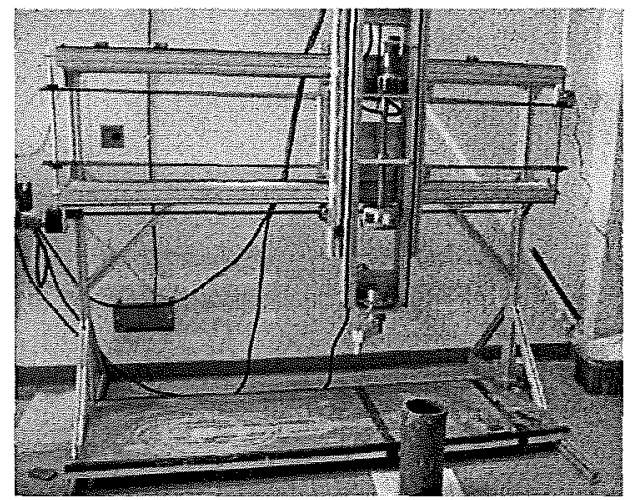

(a) 2-D Concrete Extrusion System.

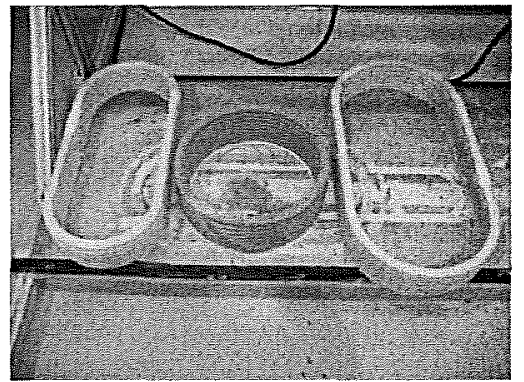

(c) Typical 2-D Concrete Extrusion Shapes.

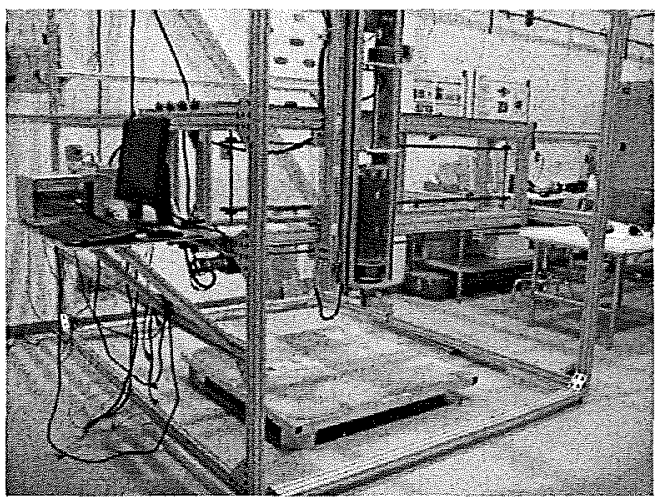

(b) 3-D Concrete Extrusion System.

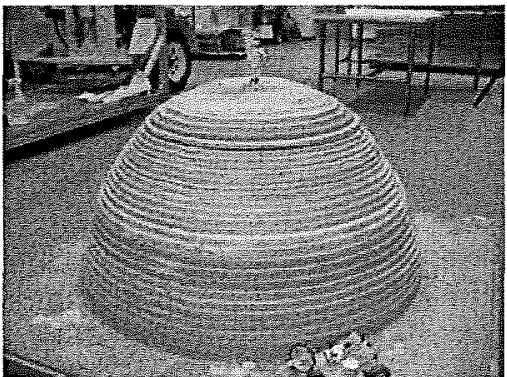

(d) 3-D Concrete Extrusion with Interior Walls,

FIGURE 3. Contour Crafting Extruded Concrete System. 
MSFC has also been evaluating the use of inflatable concrete domes. By sandwiching a layer of "wet" concrete and expandable metal coils between two hermetic liners and then pressurizing underneath the lower liner, an inflatable concrete dome, with metal or glass reinforcement, can be developed. After the concrete sets, the liners can either be left in place or removed, depending upon the application. This technology also lends itself very well to automation. Developed by architect Dante Bini (Bini, 1967), over 1,600 of these "Binishells" have been developed worldwide. In both of these technologies, reinforcement of the concrete can be provided either by adding chopped fibers of glass derived from lunar regolith, or by adding metal or glass "rebar" reinforcement (rods in tension) that are either fabricated on the Moon or Mars or provisioned. As part of this effort, MSFC has developed a temperaturedependent pressure control system that has been demonstrated to control differential pressure during inflation to $+/-$ 0.004 psid.

"Regolithbags" is a term used to describe a technique used to support or build a habitat or structure wall that consists of one or more bags designed to be filled with planetary regolith. A structure, as shown in Figure 4 (Khalili, 1989), can be made of bags brought to the Moon or Mars from Earth and filled and placed on the surface. Filled regolithbags are dense, durable, provide radiation shielding, and use readily available construction materials, but require some development in terms of sealing and containment of the very fine, very abrasive lunar regolith. MSFC has been evaluating optimization of bag material and design, potential regolith/binder compositions and automated filling and placement techniques, as shown in Figure 5.

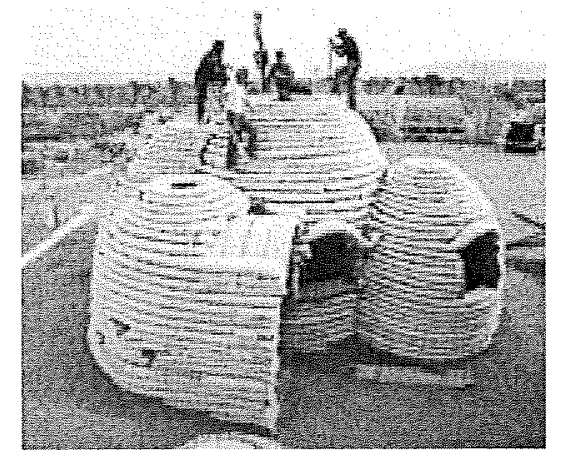

FIGURE 4. Terrestrial Application of Regolithbags to Habitat Structure.

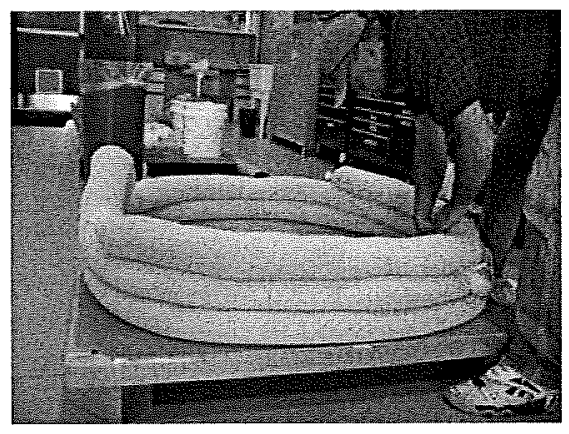

(a) Continuous Bag Structure.

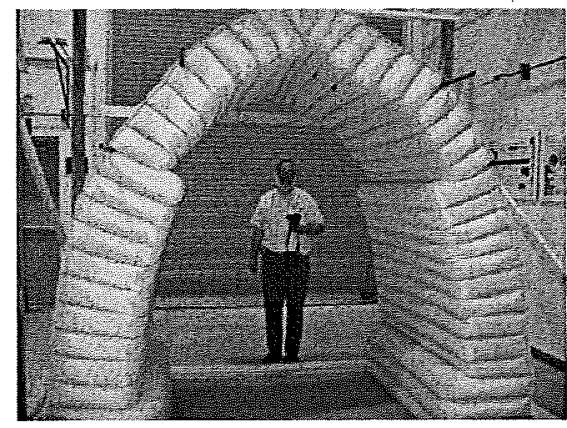

(b) Discrete Bag Structure

FIGURE 5. Initial MSFC Efforts at Regolithbag Filling, Placement, and Composition.

As part of an ongoing regolith handling and transport automation project, MSFC has developed a subscale, remotely controlled, tracked vehicle with a center dump bed and front loading scoop. This vehicle, shown in Figure 6, can be operated from a hand-held remote control or a computer interface to the remote control and is being used to evaluate dust protection techniques, mitigation of dust generation during operation, and other control functions. 


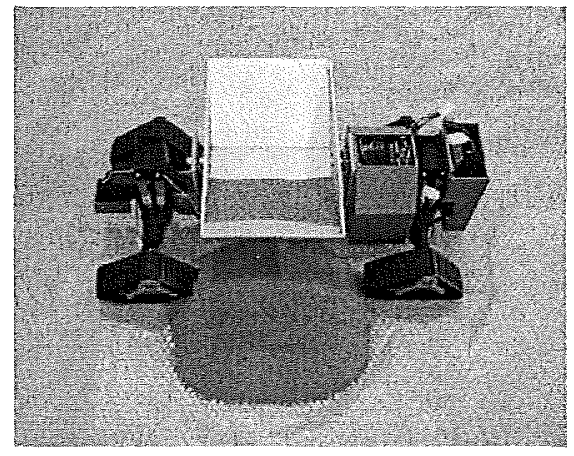

FIGURE 6. MSFC-Developed Remote Controlled Regolith Handling and Transport Vehicle.

MSFC has also been investigating several fabrication techniques for blocks made of various combinations of regolith and provisioned binders. This effort began by designing and manufacturing a set of molds to make small (approximately 0.25 -in.-cube) blocks and then using these molds to make blocks of varying compositions of JSC-1 lunar regolith simulant and various binders, including polyethylene, polyurethane, and ethylene vinyl co-polymer (EVOH). To make the polyethylene blocks, polyethylene powder was mixed with JSC-1 regolith simulant in varying compositions (polyethylene ranging from $10 \%$ to $50 \%$ by weight), manually compacted in the mold to varying pressures, and baked in the mold at $400^{\circ} \mathrm{F}$ for $1 \mathrm{hr}$. The polyurethane blocks (example shown in Figure 7) were made by mixing liquid polyurethane with JSC-1 regolith simulant in varying compositions (polyurethane ranging from $10 \%$ to $30 \%$ by weight), pouring this into the molds, and allowing it to cure for $24 \mathrm{hr}$ in the mold at ambient temperature and pressure. Block performance is now being characterized.

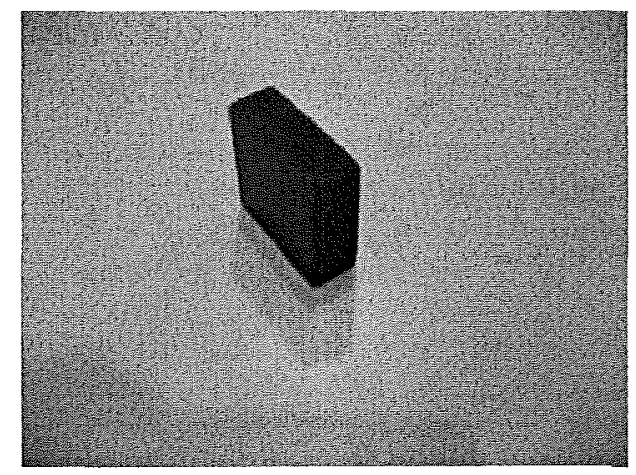

FIGURE 7. Typical Block Made from Polyurethane/JSC-1 Regolith Simulant.

Another ongoing development effort at MSFC is in the area of thin film development and processing for potential liners in existing structures or as a component of a self-supporting inflatable structure. MSFC's efforts have focused on a material called Soarnol ${ }^{\mathrm{TM}}$, a commercially available EVOH polymer known for its low oxygen permeability. Purchased as small pellets, the material has been successfully rolled and compression molded into sheets (as shown in Figure 8), compression molded into disks, and has also been evaluated for recyclability.

Last, MSFC has an ongoing effort aimed at fabrication of glass fibers and rods made of in situ materials for potential application as reinforcement for lunar concrete or for stand-alone applications as truss members, tensegrity structures, etc. Molten JSC-1 regolith simulant has been successfully melted and cast into larger diameter molds (3/8-in. and $1 / 2$-in. diameter) to demonstrate the feasibility of manufacturing "rebar," as shown in Figure 9 . Also, several hundred meters of 0.010 -in. to 0.030 -in. diameter glass fiber, shown in Figure 10, have been successfully pulled from a bath of molten JSC-1, demonstrating the ability to manufacture glass fibers. 


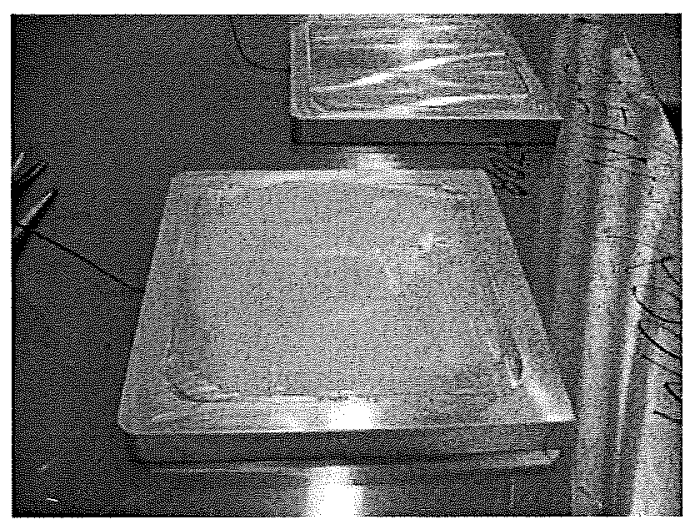

(a) Compression Mold.

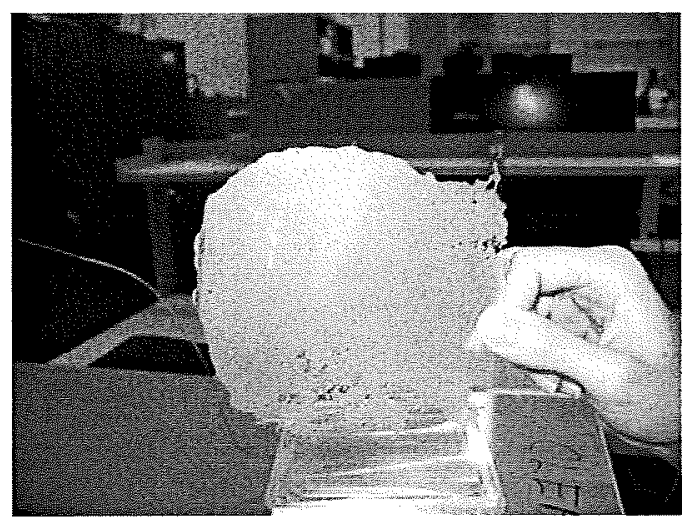

(b) First Effort at Thin Film Fabrication.

FIGURE 8. Initial Efforts at Compression Molding EVOH Pellets into Thin Films.

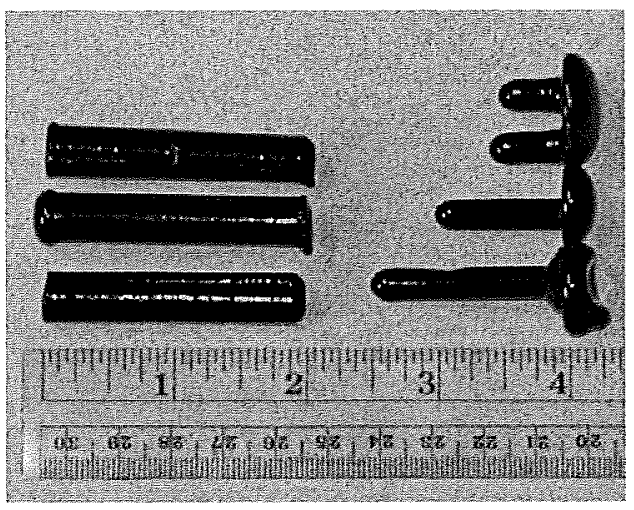

FIGURE 9. Glass "Rebar" Cast From Molten JSC-1 Regolith Simulant

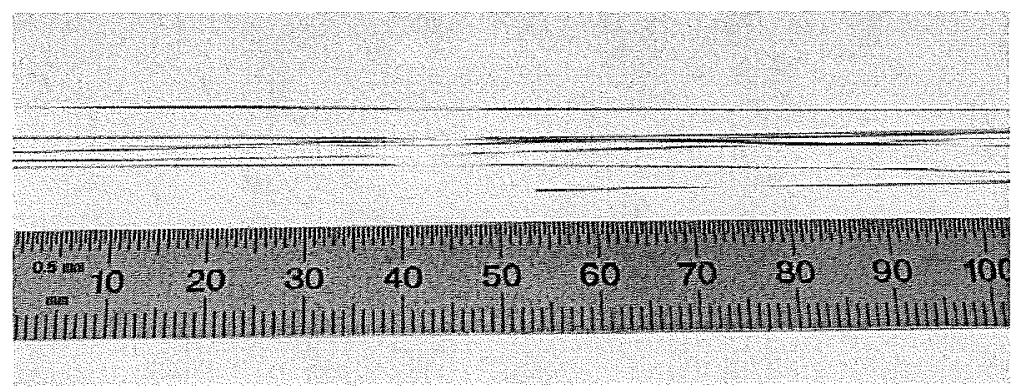

FIGURE 10. Glass Fibers Pulled from Molten JSC-1 Regolith Simulant.

\section{CONCLUSIONS}

The surface of the Moon offers a set of unique resources for development of in-situ-materials-based structures, including habitats. As part of NASA/MSFC's ISFR Habitat Structures project, a number of these resources have been and continue to be evaluated. Lunar regolith, as represented by terrestrial simulants, can be used to provide all the materials necessary to make lunar concrete, can be a major component of block manufacturing on the lunar surface, and can easily be transformed into glass elements. Future efforts will continue to characterize the performance of these materials with respect to continually developing requirements. 


\section{REFERENCES}

Bini, D., "A New Pneumatic Technique for the Construction of Thin Shells," in Proceedings of the First International Association for Shell Structures (IASS), International Colloquium on Pneumatic Structures. University of Stuttgart, Germany, 1967.

Bodiford, M.P., Fiske, M.R., McGregor, W., and Pope, R.D., "In Situ Resource-Based Lunar and Martian Habitat Structures Development at NASA/MSFC," Paper AIAA-2005-2704, in Proceedings of the AIAA $1^{\text {st }}$ Exploration Conference (CDbased), Orlando, FL, 2005.

Cohen, M. M. and Kennedy, K. J., "Habitats and Surface Construction Technology and Development Roadmap," in the Proceedings of the Government Sponsored Programs on Structures Technology (NASA CP-97-206241), edited by Noor, A. and Malone, J., 1997, pp. 75-96.

Khalili, E.N., "Lunar Structures Generated and Shielded with On-Site Materials," Journal of Aerospace Engineering, Vol. 2, No. 3, 1989.

Khoshnevis, B., Bodiford, M.P., Burks, K.B., Ethridge, E., Tucker, D., Kim, W., Toutanji, H., and Fiske, M.R., "Lunar Contour Crafting - A Novel Technique for ISRU-Based Habitat Development," in Proceedings of the $43^{\text {rd }}$ AIAA Aerospace Sciences Meeting and Exhibit (CD-based), Paper AIAA-2005-0538, 2005.

Toutanji, H., Fiske, M.R., and Bodiford, M.P., "Development of Lunar "Concrete" for Habitat Structures," in Proceedings of the $10^{\text {th }}$ ASCE Aerospace Division International Conference on Engineering, Construction and Operation in Challenging Environments: Earth \& Space 2006 (CD-based), Orlando, FL, 2006. 